\title{
WD40 Repeat 43 Mediates Cell Survival, Proliferation, Migration and Invasion via Vimentin in Colorectal Cancer
}

\section{Zijian Li}

Nanjing Drum Tower Hospital: Nanjing University Medical School Affiliated Nanjing Drum Tower Hospital https://orcid.org/0000-0001-6542-6932

\section{Min Feng}

Nanjing Drum Tower Hospital: Nanjing University Medical School Affiliated Nanjing Drum Tower Hospital

\section{Jie Zhang}

Nanjing Drum Tower Hospital: Nanjing University Medical School Affiliated Nanjing Drum Tower Hospital

\section{Xingzhou Wang}

Nanjing Drum Tower Hospital: Nanjing University Medical School Affiliated Nanjing Drum Tower Hospital

\section{En Xu}

Nanjing Drum Tower Hospital: Nanjing University Medical School Affiliated Nanjing Drum Tower Hospital

\section{Chao Wang}

Nanjing Drum Tower Hospital: Nanjing University Medical School Affiliated Nanjing Drum Tower Hospital

\section{Fengcen Lin}

Nanjing Drum Tower Hospital: Nanjing University Medical School Affiliated Nanjing Drum Tower Hospital

\section{Zhi Yang}

Nanjing Drum Tower Hospital: Nanjing University Medical School Affiliated Nanjing Drum Tower Hospital

\section{Heng Yu}

Nanjing Drum Tower Hospital: Nanjing University Medical School Affiliated Nanjing Drum Tower Hospital

\section{Wenxian Guan ( $\nabla$ wenxian_guan@sina.com )}

Nanjing Drum Tower Hospital: Nanjing University Medical School Affiliated Nanjing Drum Tower Hospital

\section{Hao Wang}


Nanjing Drum Tower Hospital: Nanjing University Medical School Affiliated Nanjing Drum Tower Hospital

\section{Research Article}

Keywords: WDR43, VIM, CRC, Biomarker

Posted Date: March 5th, 2021

DOI: https://doi.org/10.21203/rs.3.rs-269248/v1

License: (c) (1) This work is licensed under a Creative Commons Attribution 4.0 International License. Read Full License

Version of Record: A version of this preprint was published at Cancer Cell International on August 9th, 2021. See the published version at https://doi.org/10.1186/s12935-021-02109-1. 


\section{Abstract}

\section{Background}

WD40 repeat (WDR)43 is an RNA-binding protein that belongs to the WDR domain protein family. Its biological function is largely unclear, particularly in colorectal cancer (CRC).

\section{Methods}

In the present study, we searched the TCGA database and found the correlation between WDR43 and CRC. Subsequently,the high expression of WDR43 in human clinical samples of CRC was validated and we further examined the biological functions of it in CRC cells. Finally,we explored potential downstream proteins or pathways and established subcutaneous xenograft model to verify our findings.

Results

Immunohistochemistry of 16 patient specimens confirmed that the expression of WDR43 was elevated in CRC. WDR43 knockdown was shown to increase apoptosis and inhibit the proliferation, migration and invasion of CRC cells in vitro and reduce tumorigenesis in animal models. In addition, it was found that WDR43 knockdown inhibited vimentin (VIM) expression in CRC cells and overexpression of VIM can partially reverse the effects of WDR43 both in vitro and in vivo.

\section{Conclusion}

In conclusion, the role of WDR43 in the occurrence and development of CRC was investigated in the present study. WDR43 may serve as a valuable biomarker and provide new options for the diagnosis and treatment of colorectal cancer.

\section{Introduction}

Colorectal cancer (CRC) accounts for $10 \%$ of the total cases of cancer and cancer-related mortality worldwide per annum (1). Particularly in developing countries, the incidence of CRC is gradually increasing (2). In China, despite the widespread use of early screening for individuals with a high-risk family history, most patients are diagnosed at the late stage of the disease and cannot benefit from surgical treatment (3). Patients with distant metastases generally have a relatively poor prognosis, due to accelerated tumor spreading and resistance to chemotherapy and radiotherapy, which makes CRC a difficult clinical challenge $(4,5)$. Therefore, it is very important to identify novel targets for the treatment and diagnosis of CRC. The WD40 repeat (WDR) domain is a domain composed of multiple $\beta$-helical structures. It is usually used as a scaffold for protein interaction and provides a platform for the assembly of multi-protein complexes (6). Proteins containing the WDR domain participate in a wide range of cellular networks, such as signal transduction, transcriptional regulation, cell cycle control, cytoskeleton assembly and chromatin modification, many of which are associated with human diseases (7). The WDR43 protein belongs to the protein family containing the WDR domain. It is usually involved in 
the assembly of small-subunit processome as a conserved component, and mediates the transcription and processing of small-subunit ribosomal RNA (18S rRNA), which plays a key role in the biogenesis of ribosomes $(8,9)$. Studies have shown that WDR43 can suspend the release of Pol II and promote transcription extension to regulate the high-level transcription and translation process in the pluripotency of embryonic stem cells (10). WDR43 mutations were not only found to cause defects in craniofacial development in zebrafish (11), but were also associated with the occurrence of human estrogen receptornegative breast cancer (12). These findings suggested that WDR43 plays a key role in cell proliferation and may be involved in the occurrence and development of cancer. However, the specific role of WDR43 in CRC remains unclear; therefore, the present study was designed to explore the impact of WDR43 on the biological behavior of CRC and determine whether it may be of value as a new target for the diagnosis and treatment of CRC.

\section{Materials And Methods}

Immunochemistry. Colorectal multi-tumor tissue microarrays (TMAs) were purchased from Genechem Co., Ltd. The TMAs were blocked with $5 \%$ goat serum dissolved in PBS for 60 min and subsequently incubated with Rabbit polyclonal anti-WDR43 antibody (cat no. ab174906; dilution, 1:50; Abcam) overnight at $4^{\circ} \mathrm{C}$. Following staining, the samples were assessed by a senior pathologist, and a blinded manner were taken to record the immunohistochemical results. The final score of each microarray was based on the proportion of positively colored cells $(0,0-5 \% ; 1,6-25 \% ; 2,26-50 \% ; 3,51-75 \%$; and $4,76-$ $100 \%$ ) and staining intensity ( 0 , no staining; 1 , thin staining; 2 , middling staining; and 3 , strong staining). Low expression was defined as a final score of $<6$, and high as a final score of $\geq 6$. The present study was authorized by the Ethics Committee of the Nanjing Drum Tower Hospital.

Cell culture. The DLD-1 CRC cell line was purchased from Procell Life Science \& Technology Co., Ltd., and the HCT116 CRC cell line from Nanjing KeyGen Biotech Co., Ltd.. Both cell lines were STR-authenticated. Tumor cells were incubated at $37^{\circ} \mathrm{C}, 5 \% \mathrm{CO} 2$, DMEM or McCoy's $5 \mathrm{~A}$ medium (Thermo Fisher Scientific, Inc.) containing $10 \%$ fetal bovine serum (Gibco, USA) for DLD-1 and HCT116 cells, respectively. The medium was renewed every 3 days and the cells were passaged when the cell density reached $80-90 \%$.

Lentiviral transfection. The shRNA plasmid vector was constructed for lentiviral (GeneChem, Inc.) infection to stably knockdown WDR43. The whole transfection procedure was then performed following the manufacturer's instructions. Puromycin was used to eliminate the untransfected cells 3 days after transfection. The transfection of the surviving cells was confirmed by RT-qPCR.

RNA extraction and RT-qPCR. Total RNA of cells was extracted using TRIzol® reagent (Merck KGaA) and detected by BioDrop $\mu$ LITE+ (Biochrom, Ltd.). RT was then performed using HiScript III RT SuperMix for qPCR (Vazyme Biotech Co., Ltd.) to synthesize cDNA. Next, RT-qPCR was executed on a ViiA ${ }^{\text {TM }} 7$ Real-Time PCR System (Thermo Fisher Scientific, Inc.) using the comparative threshold cycle (2- $\Delta \Delta C \mathrm{Ct})$ method.

Target gene expression at the mRNA level was relatively quantified using GAPDH as the internal reference gene. Following is primer sequences : WDR43, 5'-CCAGGGCTTAGAAAGTAACGA-3' forward and 5'- 
TCAGGCAACGTGGACAGGTAT-3' reverse (final product size, 228 bp); GAPDH, 5'-

TGACTTCAACAGCGACACCCA-3' forward and, 5'-CACCCTGTTGCTGTAGCCAAA-3' reverse (final product size, 121 bp). Acquired data were finally analyzed by GraphPad Prism 7.0 (GraphPad Software, Inc.).

Protein extraction and immunoblotting. Proteins were extracted from cells with $100 \mu$ l RIPA buffer containing 1:100 Complete $^{\text {TM }}$ Protease Inhibitor Cocktail (Roche Diagnostics). Following lysing and centrifugation, the protein-containing supernatant was mixed with 5X SDS-PAGE loading buffer (1:4 ratio; Biosharp Life Sciences) and boiled for 5 min to denaturate the protein. BCA assay (Nanjing KeyGen Biotech Co., Ltd.) was used for protein quantification. Equal amounts of proteins $(15 \mu \mathrm{g})$ from each sample were loaded in 10\% gel (PG112, epizyme) and transferred on PVDF membrane (10600021, GE Healthcare). The membranes were then cut into stripes and blocked with 10\% skim milk (1172GR500, Biofroxx). Then the stripes were incubated with primary antibodies against WDR43 (cat. no. ab174906; dilution, 1:300; Abcam) and the internal reference protein GAPDH (cat. no. sc-32233; dilution, 1:2000), followed by incubation with secondary antibodies for two hours at room temperature (7074S, dilution, 1:4000, Cell-Signaling Technology, Inc.). 20X LumiGLO® Reagent and 20X peroxide (7003S; CellSignaling Technology, Inc.) were used in development following the instruction, and the bands were imaged using a Tanon 5200 Chemiluminescent Imaging System (Tanon Science \& Technology Co., Ltd).

MTT and Celigo assays. Cells that had received different treatments were harvested using trypsin with 0.25\% EDTA (Thermo Fisher Scientific, Inc.) and counted using Countstar® BioTech Automated Cell Counter (Countstar). The cells were then seeded into 96-well plates (1.5x103 cells/well). For Celigo assays, the number of cells was counted each day using the Celigo Image Cytometer (Nexcelom Bioscience LLC). For MTT assays, the cells were then incubated respectively for 24, 48, 72, 96 and $120 \mathrm{~h}$. MTT reagent ( $20 \mu \mathrm{l} ; 5 \mathrm{mg} / \mathrm{ml}$; Genview) was added $4 \mathrm{~h}$ before the incubation was terminated. Once precipitates became visible, the supernatants were carefully discarded and $100 \mu$ dimethyl sulfoxide was added for 2-5 min to dissolve the precipitates. The absorbance value of each well was detected by a microplate reader at $490 \mathrm{~nm}$. Each experiment was repeated at least three times.

Colony formation assay. Transfected cells were cultivated into each well of a 6 -well culture plate $(1.5 \times 103$ cells/well) and cultured for 8 days, with renewal of the medium every 3 days during the incubation. The cells were then washed with $1 \mathrm{ml}$ PBS at a time, fixed by $1 \mathrm{ml} 4 \%$ paraformaldehyde for $25 \mathrm{~min}$ at room temperature, and rewashed once with $1 \mathrm{ml}$ PBS. Then, cells were colored with $1 \mathrm{ml}$ crystal violet solution (Beyotime, C0121) (Sangon Biotech Co., Ltd.) for $15 \mathrm{~min}$ at room temperature and then washed with $\mathrm{ddH} 2 \mathrm{O}$ several times. The plate was air-dried and photographed with a digital camera to count the number of colonies containing $>50$ cells. Following formula was used to calculat the colony formation efficiency: Colony formation efficiency = (number of colonies/number of inoculated cells) $\times 100 \%$.

Flow cytometry. Flow cytometry was used to analyze the apoptotic rate of tumor cells that had received different treatments. Annexin V Apoptosis Detection Kit II (556570; BD Biosciences) was used for incubation with the collected cells to detect the apoptotic rate. The results were detected and obtained by 
BD AccuriTM C6 Plus cell analyzers (BD Biosciences). FlowJo 10.4 (FlowJo, LLC) was used to analyze the final results.

Cell migration and invasion. To evaluate the effect of WDR43 on migration and invasion of cells, Transwell assays were performed. Briefly, Transwell ${ }^{\circledR}$ chambers with an 8.0- $\mu \mathrm{m}$ Pore Polycarbonate Membrane (cat. no. 3422; Corning) were placed in a 24-well plate, with serum-free culture medium in the upper chamber and medium having $10 \%$ fetal bovine serum in the lower chamber; tumor cells $(1 \times 105$ cells) were placed into the upper chamber. Following $24 \mathrm{~h}$ of cultivation, $4 \%$ paraformaldehyde was used to fix the cells for $15 \mathrm{~min}$ and then cells were colored with crystal violet solution (Beyotime, C0121) for 20 min. Following washing with PBS and wiping, the final results were photographed under Inverted Microscope Solution DMi8 S Platform (Leica Microsystems, Inc.) and analyzed by Leica Application Suite $X$ (Leica Microsystems, Inc.). For cell invasion assays, the chambers were coated with Corning ${ }^{\circledR}$ Matrigel ${ }^{\circledR}$ Basement Membrane Matrix (cat. no. 356324; Corning) and incubated for 30 min; the rest of the procedure was coincident as that for the migration assay.

In vivo experiments. Male BALB/c nude mice aged 4-6 weeks were provided by the Nanjing Medical University. Tumor cells were digested with trypsin and adjusted to $1 \times 10^{7}$ cells $/ \mathrm{ml}$, and then subcutaneously injected into the back of mice. Each group contained a minimum of 5 mice. Mice were sacrificed 4 weeks after treatment, and tumors were subsequently resected to assess weight and volume.

Statistical analysis. All experiments were repeated at least three times. GraphPad Prism 7.0 (GraphPad Software) was used to analyze the data and determine the significance of the differences. For categorical data, student t-test was performed for comparisons between two groups, and one-way ANOVA for comparisons among multiple groups. $p<0.05$ was considered statistically significant.

\section{Results}

WDR43 is associated with the stage and tissue subtypes of CRC. Following a search of the TCGA database, the expression of WDR43 was found to be increased in CRC, as compared to that in canceradjacent normal colon tissue (Fig. 1a). The upregulation of WDR43 was found to be associated with the stage (Fig. 1b) and subtype (Fig. 1c) of CRC, but exhibited no significant correlation with prognosis (Fig. 1d).

WDR43 expression is increased in CRC tissues. Immunohistochemistry was used to examine 16 pairs of cancer specimens and normal tissues adjacent to cancer from CRC patients. The immunohistochemical score (\% of positive cells $x$ staining intensity) showed that the expression level of WDR43 in CRC tumor tissue was higher compared with that cancer-adjacent normal tissues (Fig. 2).

WDR43 is highly expressed in CRC cells. The level of WDR43 mRNA was detected in 4 CRC cell lines and the NCM460 human normal colonic epithelial cell line (Fig. 3a). As compared with NCM460, the WDR43 expression was increased in the $4 \mathrm{CRC}$ cell lines. In order to reduce contingency, two cell lines with a medium expression level were selected for subsequent functional tests. Lentivirus was used to obtain 
DLD-1 and HCT116 cells with stably knocked down WDR43. RT-qPCR and western blotting (WB) were performed to evaluate WDR43 knockdown and confirm the knockdown efficiency in the two cell lines (Fig. 3b and c).

WDR43 knockdown inhibits the proliferation and colony formation of CRC cells in vitro. Following WDR43 knockdown, the proliferative ability of the DLD-1 and HCT116 cell lines was examined. The cells were continuously counted by MTT(Additional file 1: Table S1) and Celigo(Additional file 1: Table S2) experiments for 5 days. It was found that, following WDR43 knockdown, the proliferative rate of DLD-1 and HCT116 cells was significantly inhibited (Fig. 4a and b), suggesting that low WDR43 expression inhibits CRC cell proliferation. The colony formation experiment also confirmed this result (Fig. 4c).

WDR43 knockdown promotes apoptosis in CRC cells. Flow cytometry was performed to investigate whether WDR43 promotes CRC cell proliferation by regulating apoptosis. It was shown that changes in WDR43 levels affect the apoptotic rate. As compared with the control group, the number of apoptotic cells increased in the two cell lines with a decreased WDR43 gene expression (Fig. 5,Additional file 2: Table S3 and S4).

WDR43 knockdown inhibits migration and invasion in CRC cells. Transwell assay was performed to investigate the effect of WDR43 knockdown on the invasion and migration of CRC cells. After 24 and 48 $\mathrm{h}$ of viral infection, the migrating and invasive cells were counted, and it was found that WDR43 knockdown inhibited the migration and invasion of CRC cells (Fig. 6a and b).

WDR43 knockdown suppresses vimentin (VIM) expression. In order to explore the mechanism underlying the effect of WDR43 on the proliferation and migration of CRC cells, the downstream functional proteins that may be regulated by WDR43 were examined. Following the lentiviral knockdown of WDR43 in DLD-1 cells, WB was used to detect the expression of several proteins associated with tumor cell proliferation and migration, including N-cadherin, E-cadherin, Slug, Snail, VIM, MYC, fibronectin 1 and matrix metalloproteinase-2, and classic signaling pathway proteins and their phosphorylated forms, including $\beta$ catenin, p- $\beta$-catenin, extracellular signal-regulated kinase (ERK)1/2, p-ERK1/2, P-38, p-P38, protein kinase B (Akt), p-Akt, nuclear factor-KB (NF-KB) p65, p-NF-кB p65, mechanistic target of rapamycin (mTOR) and p-mTOR. The results showed that, following WDR43 knockdown, the expression of VIM was significantly reduced (Fig. 7).

VIM overexpression partially restores the proliferative and migratory ability of CRC cells following WDR43 knockdown. In order to confirm the function of VIM, lentivirus was used to simultaneously knock down WDR43 and overexpress VIM in DLD-1 cells. RT-qPCR was used to test the transfection efficiency and ensure that stably transfected strains were obtained (Fig. 8a). MTT and Celigo experiments were used to test the proliferative ability of the cells and found that, following VIM overexpression, the suppression of cell proliferation caused by WDR43 knockdown was significantly recovered (Fig. 8b and c,Additional file 3: Table S5 and S6). Transwell assay results also showed that, as compared with WDR43 knockdown, the migration and invasion of DLD-1 cells with WDR43 knockdown and VIM overexpression were increased (Fig. 8d). 
WDR43 knockdown inhibits the proliferation of CRC cells in vivo. In order to study whether WDR43 plays a same role in the proliferation of CRC in vivo, a subcutaneous xenograft model of Balb/c nude mice was established using DLD-1 cells. As compared with the control group, the tumor volume and weight of mice in the WDR43 knockdown group was reduced, while the tumor volume and weight of mice with both WDR43 knockdown and VIM overexpression were significantly recovered (Fig. 9). Therefore, a low WDR43 expression decreases the growth of tumor in vivo, which was accord with our research in vitro and clinical discoveries.

\section{Discussion}

In the present study, high WDR43 expression level was confirmed in CRC cells and tissues, and it was found that WDR43 promotes the proliferation, migratory, invasive and anti-apoptotic ability of CRC cells. At the same time, WDR43 was also found to regulate the expression of downstream functional protein VIM. These results preliminarily illustrated the role of WDR43 in the development of CRC, and may provide a valuable reference for further expanding the application of WDR43 in tumor research.

As one of the most plentiful domains of protein-protein reciprocity in the proteome of mankind, the WDR domain has been shown to participate in a wide range of cellular networks (7). This domain has been associated with several human diseases $(6,7)$. Particularly in the field of oncology, certain proteins containing WDR domains may be used as effective targets for cancer treatment (13). Grebien et al (14) used the specific WDR5 antagonist OICR-9429 to disrupt the MLL1-WDR5 interaction, thereby killing p30expressing acute myeloid leukemia cells. O'Bryant et al (15) found that WDR77 knockdown can inhibit E2F3 activation and enhance TGF $\beta$ signaling, abolishing the prostate tumorigenesis induced by PTEN deletion. Based on the correlation between WDR protein and cancer, CRC-related genes were screened out by genome-wide gene expression profiling (16), and WDR43 was selected to explore its impact on tumor biological functions. In the present study, the inhibitory effect of WDR43 knockdown on CRC in vivo and in vitro was observed. Similarly, it was found by Zhao et al (17) that the loss of WDR43 in zebrafish caused early developmental defects in various tissues. In addition, Fujimura et al (18) proved that the nucleolar protein 11 (NOL11) forms a protein complex called NWC with WDR43 and Cirhin, and participates in the process of chromosome mitosis by promoting the enrichment of Aurora B on the centromere. The present study also reported an effect of WDR43 on the migratory ability of CRC cells, an effect that appeared to be achieved through the regulation of VIM, which was manifested as a significant decrease in VIM expression following WDR43 knockdown. Similarly, in the study by Zhu et al (19), miR34a inhibited the expression of VIM and reduced the migratory and invasive ability of CRC cells. As the main component of the intermediate filament protein family, VIM has the ability to maintain cell integrity and enable resistance to stress (20). A number of studies have confirmed that increased expression of VIM is associated with tumor metastasis and invasiveness (21), and is considered to be a classic marker of epithelial-mesenchymal transition (EMT) (22). Although the effect of overexpression of VIM on the proliferation and migration of CRC cells was confirmed, the evidence obtained was insufficient to support the involvement of EMT in the WDR43 regulatory network, and further research is necessary. Finally, with regards to the failure of this study to screen out the specific signaling pathways that WDR43 may directly 
regulate, the possible explanation is that WDR43 may play a more basic role in nucleic acid metabolism, which requires the support of bioinformatics and subsequent deeper mining.

\section{Conclusion}

In conclusion, to the best of our knowledge, this study is the first to report that WDR43 knockdown inhibits the proliferative, migratory, invasive and anti-apoptotic ability of CRC cells. These findings have enriched our understanding of and research on WDR domains, providing a valuable reference for the diagnosis and treatment of CRC in the future.

\section{Abbreviations}

WDR43: WD40 repeat 43

CRC: colorectal cancer

VIM: vimentin

18S rRNA: small-subunit ribosomal RNA

TMAs: tissue microarrays

RT-qPCR: Reverse transcription quantitative polymerase chain reaction

WB: western blotting

CDH1: E-cadherin

$\mathrm{CDH}$ : N-cadherin

FN1: fibronectin 1

(p-)AKT: (phosphorylated-)protein kinase B

MMP-2: matrix metalloproteinase-2

(p-)mTOR: (phosphorylated-)mechanistic target of rapamycin

(p-)NF-kB p65: (phosphorylated-)nuclear factor-kB p65

(p-)ERK1/2: (phosphorylated-)extracellular signal-regulated kinase1/2

VIM: vimentin

NC: negative control 
KD: WDR43 knockdown

OE: VIM overexpression

EMT: epithelial-mesenchymal transition

\section{Declarations}

\section{Availability of data and material}

The data sets analysed during the current study are available in the TCGA repository(http://ualcan.path.uab.edu/cgi-bin/TCGAExResultNew2.pl?genenam=WDR43\&ctype=COAD).

\section{Acknowledgements}

Not applicable.

\section{Funding}

This work was supported by the National Nature Science Foundation of China (grant no. 81670561), the Natural Science Foundation of Jiangsu Province (grant no. QNRC2016022) and the Fundamental Research Funds for the Central Universities (grant no. 021414380445).

\section{Contributions}

WXG and HW supervised the project and contributed to the design and interpretation of all experiments. ZJL,MF and JZ contributed to the design, conduct, and interpretation of all experiments.FCL, ZY and HY contributed to experimental studies. XZW,EX and CW contributed to data analysis. All authors read and approved the final manuscript.

\section{Ethics declarations}

\section{Ethics approval and consent to participate}

This study was approved by the Drum Tower Clinical Medical College of Nanjing Medical University.

\section{Consent for publication}

All authors read and approved the final manuscript.

\section{Competing interests}

The authors declared no financial conflict of interest.

\section{References}


1. BRAY F, FERLAY J, SOERJOMATARAM I, et al. Global cancer statistics 2018: GLOBOCAN estimates of incidence and mortality worldwide for 36 cancers in 185 countries [J]. CA: a cancer journal for clinicians, 2018, 68(6): 394-424.

2. DEKKER E, TANIS P J, VLEUGELS J L A, et al. Colorectal cancer [J]. Lancet (London, England), 2019, 394(10207): 1467-80.

3. CHEN W, ZHENG R, BAADE P D, et al. Cancer statistics in China, 2015 [J]. CA: a cancer journal for clinicians, 2016, 66(2): 115-32.

4. ALBERTS S R, HORVATH W L, STERNFELD W C, et al. Oxaliplatin, fluorouracil, and leucovorin for patients with unresectable liver-only metastases from colorectal cancer: a North Central Cancer Treatment Group phase II study [J]. Journal of clinical oncology : official journal of the American Society of Clinical Oncology, 2005, 23(36): 9243-9.

5. MEYERHARDT J A, MAYER R J. Systemic therapy for colorectal cancer [J]. The New England journal of medicine, 2005, 352(5): 476-87.

6. JAIN B P, PANDEY S. WD40 Repeat Proteins: Signalling Scaffold with Diverse Functions [J]. The protein journal, 2018, 37(5): 391-406.

7. SCHAPIRA M, TYERS $M$, TORRENT $M$, et al. WD40 repeat domain proteins: a novel target class? [J]. Nature reviews Drug discovery, 2017, 16(11): 773-86.

8. SONDALLE S B, BASERGA S J, YELICK P C. The Contributions of the Ribosome Biogenesis Protein Utp5/WDR43 to Craniofacial Development [J]. Journal of dental research, 2016, 95(11): 1214-20.

9. PEñA C, HURT E, PANSE V G. Eukaryotic ribosome assembly, transport and quality control [J]. Nature structural \& molecular biology, 2017, 24(9): 689-99.

10. BI X, XU Y, LI T, et al. RNA Targets Ribogenesis Factor WDR43 to Chromatin for Transcription and Pluripotency Control [J]. Molecular cell, 2019, 75(1): 102-16.e9.

11. PARANT J M, GEORGE S A, PRYOR R, et al. A rapid and efficient method of genotyping zebrafish mutants [J]. Developmental dynamics : an official publication of the American Association of Anatomists, 2009, 238(12): 3168-74.

12. COUCH F J, KUCHENBAECKER K B, MICHAILIDOU K, et al. Identification of four novel susceptibility loci for oestrogen receptor negative breast cancer [J]. Nature communications, 2016, 7(11375.

13. SONG R, WANG Z D, SCHAPIRA M. Disease Association and Druggability of WD40 Repeat Proteins [J]. Journal of proteome research, 2017, 16(10): 3766-73.

14. GREBIEN F, VEDADI M, GETLIK M, et al. Pharmacological targeting of the Wdr5-MLL interaction in C/EBPa N-terminal leukemia [J]. Nature chemical biology, 2015, 11(8): 571-8.

15. O'BRYANT D, WANG Z. The essential role of WD repeat domain 77 in prostate tumor initiation induced by Pten loss [J]. Oncogene, 2018, 37(30): 4151-63.

16. SUN L C, QIAN HX. Screening for implicated genes in colorectal cancer using whole-genome gene expression profiling [J]. Molecular medicine reports, 2018, 17(6): 8260-8. 
17. ZHAO C, ANDREEVA V, GIBERT Y, et al. Tissue specific roles for the ribosome biogenesis factor Wdr43 in zebrafish development [J]. PLoS genetics, 2014, 10(1): e1004074.

18. FUJIMURA A, HAYASHI Y, KATO $K$, et al. Identification of a novel nucleolar protein complex required for mitotic chromosome segregation through centromeric accumulation of Aurora B [J]. Nucleic acids research, 2020, 48(12): 6583-96.

19. ZHU W, LONG J L, YIN Y T, et al. MicroRNA-34a suppresses the invasion and migration of colorectal cancer cells by enhancing EGR1 and inhibiting vimentin [J]. Experimental and therapeutic medicine, 2019, 18(4): 2459-66.

20. SHARMA P, ALSHARIF S, FALLATAH A, et al. Intermediate Filaments as Effectors of Cancer Development and Metastasis: A Focus on Keratins, Vimentin, and Nestin [J]. Cells, 2019, 8(5):

21. SATELLI A, LI S. Vimentin in cancer and its potential as a molecular target for cancer therapy [J]. Cellular and molecular life sciences : CMLS, 2011, 68(18): 3033-46.

22. THIERY J P. Epithelial-mesenchymal transitions in tumour progression [J]. Nature reviews Cancer, 2002, 2(6): 442-54.

\section{Figures}


a

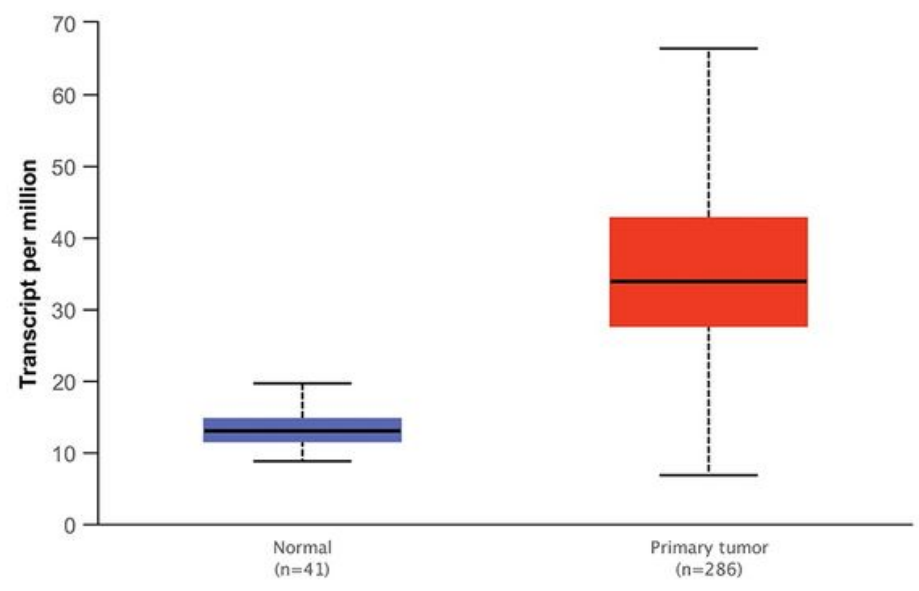

c

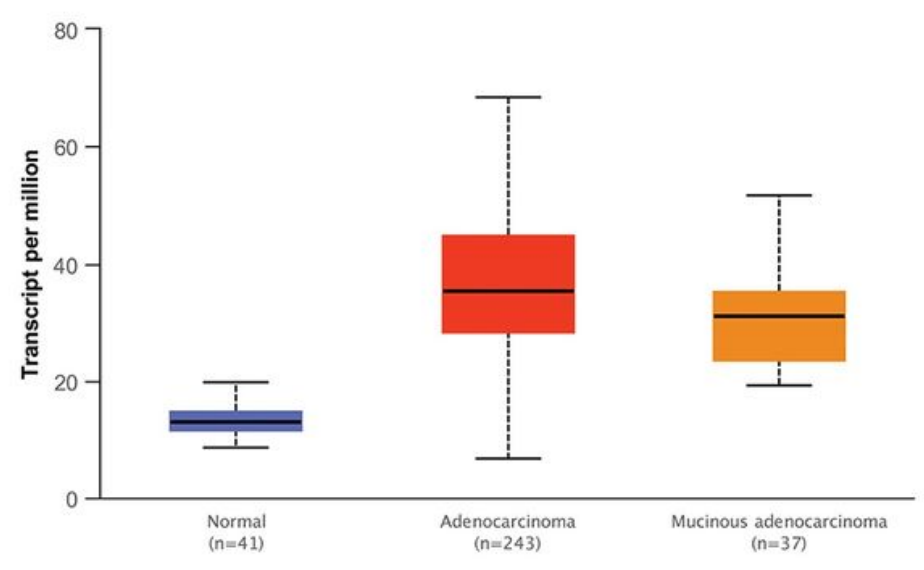

b

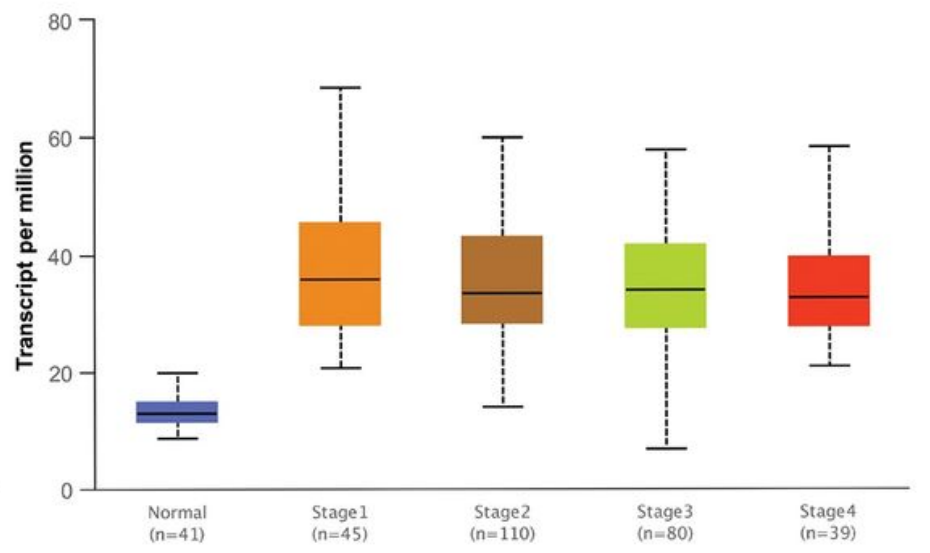

$\mathrm{d}_{\mathrm{s}, \mathrm{d}}$

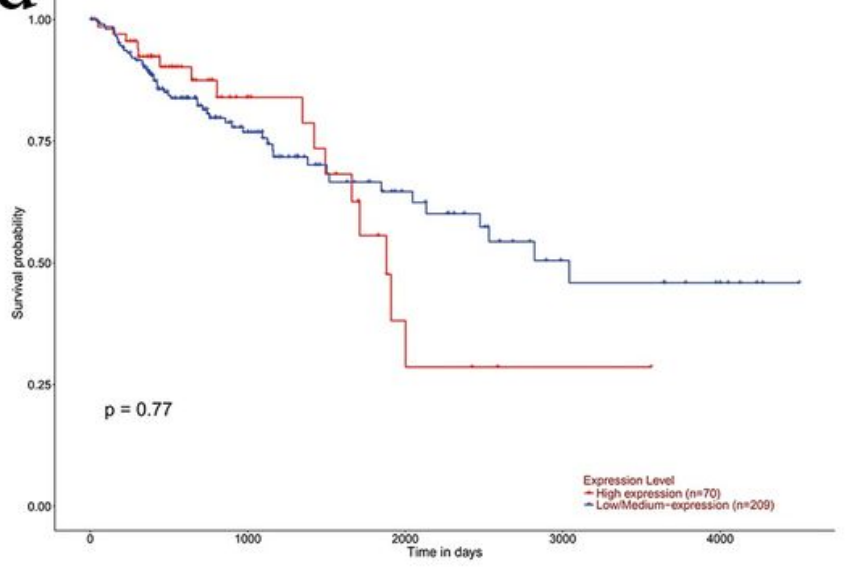

Figure 1

WDR43 is associated with the stage and subtype of CRC. TCGA suggested that WDR43 is (a) upregulated and correlated with (b) disease stage and (c) tumor subtype, but not with (d) prognosis. WDR, WD40 repeat; CRC, colorectal cancer; TCGA, The Cancer Genome Atlas. 


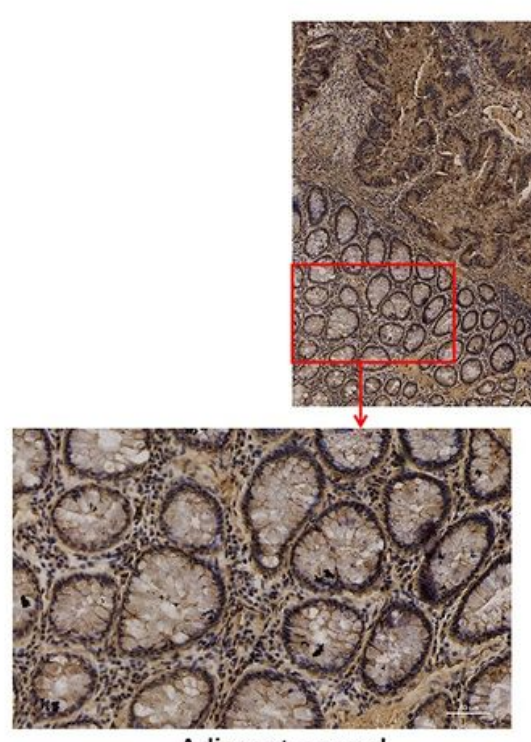

Adjacent normal

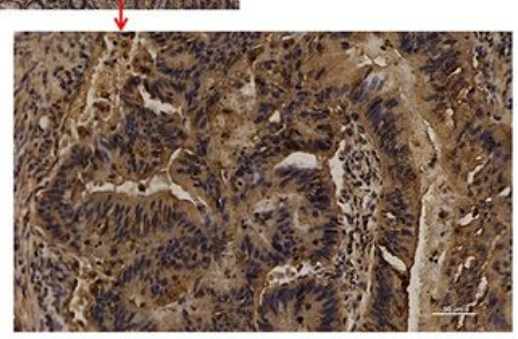

Colon cancer

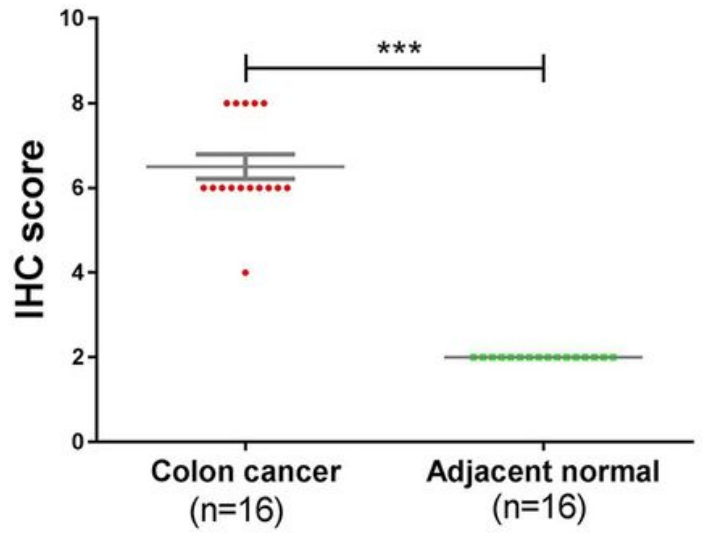

\section{Figure 2}

WDR43 expression is increased in CRC tissues. Representative images of immunohistochemical staining for WDR43 in tissue microarrays. Magnification, x50. WDR, WD40 repeat; CRC, colorectal cancer.
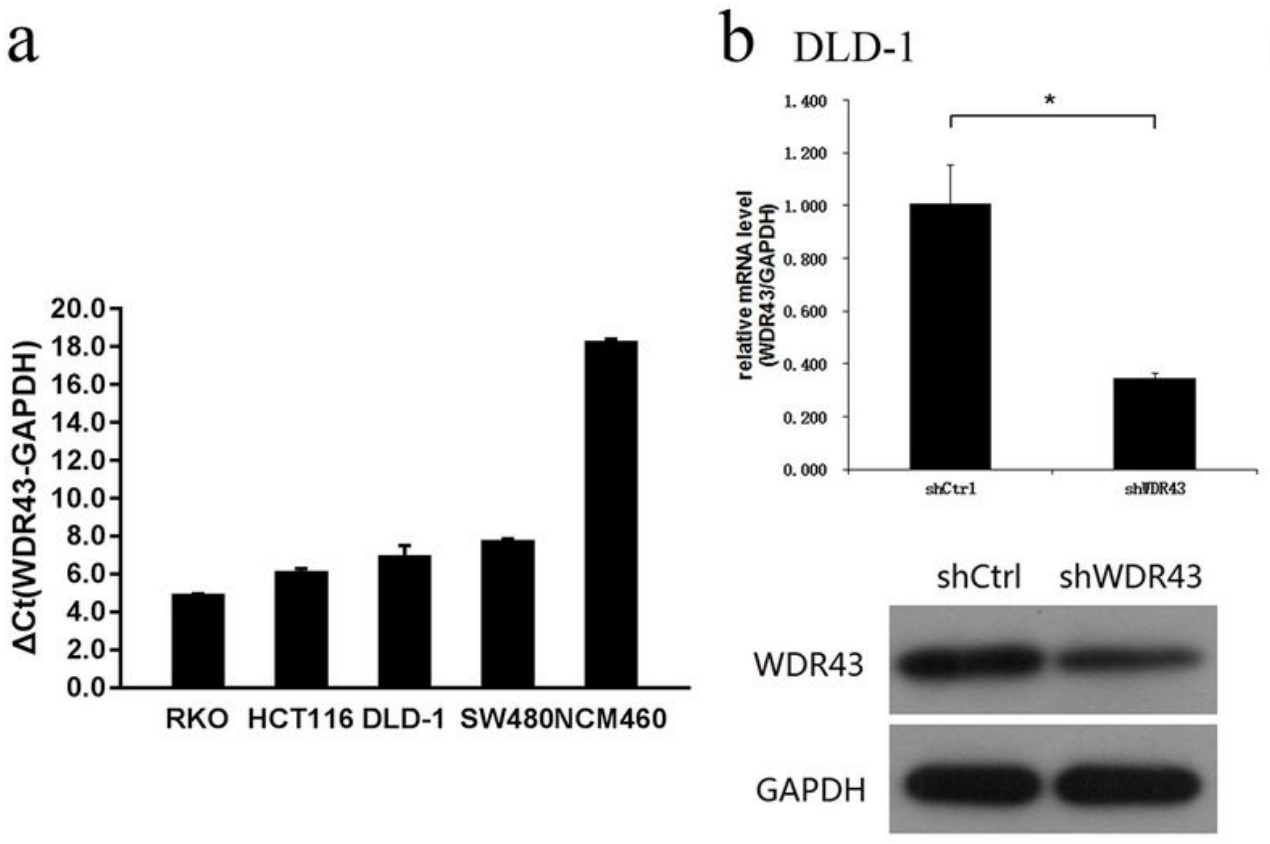

C HCT116
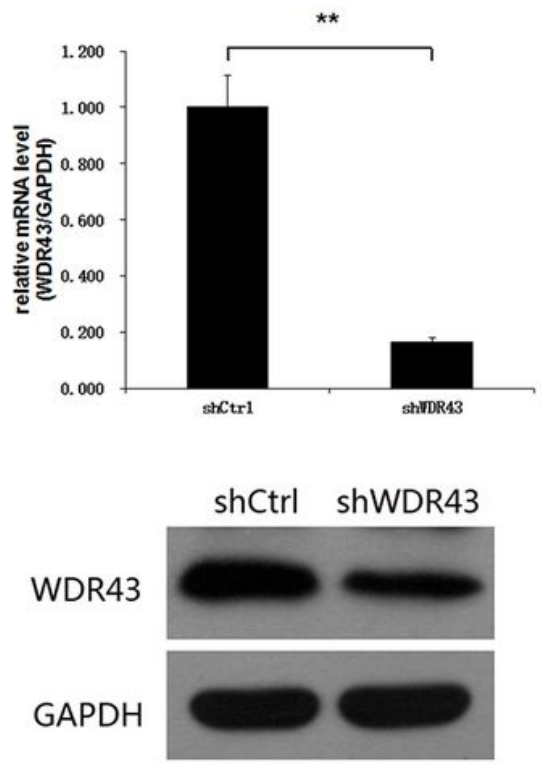

\section{Figure 3}

WDR43 is highly expressed in CRC cells. (a) WDR43 mRNA expression levels in a normal colonic cell line and 4 CRC cell lines. Gene and protein levels of WDR43 in (b) DLD-1 and (c) HCT116 cells transfected with lentiviral targeting WDR43. WDR, WD40 repeat; CRC, colorectal cancer; shCtrl, negative control; shWDR43, cells transfected with lentiviral targeting WDR43. 
a DLD-1

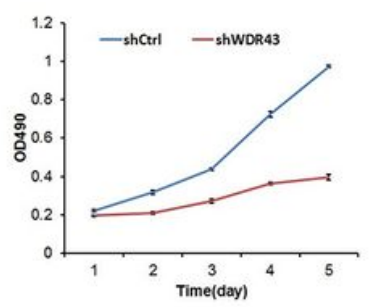

b
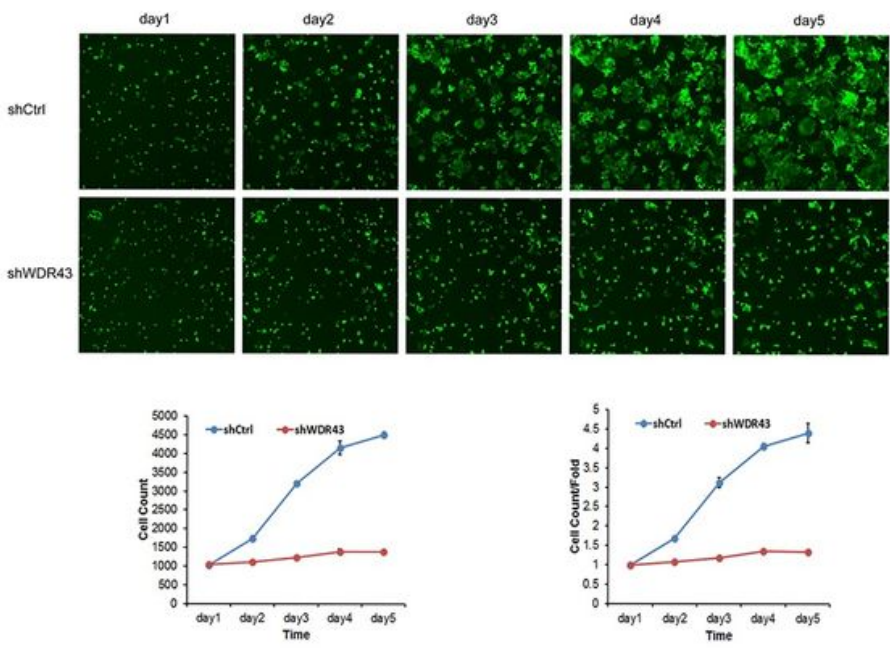

C
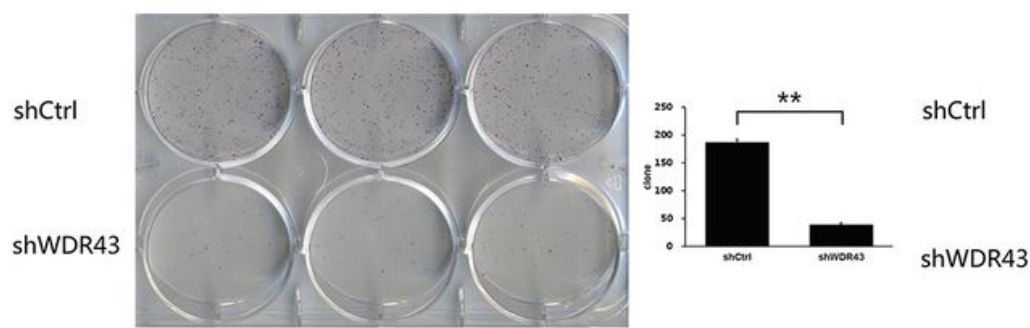

HCT116
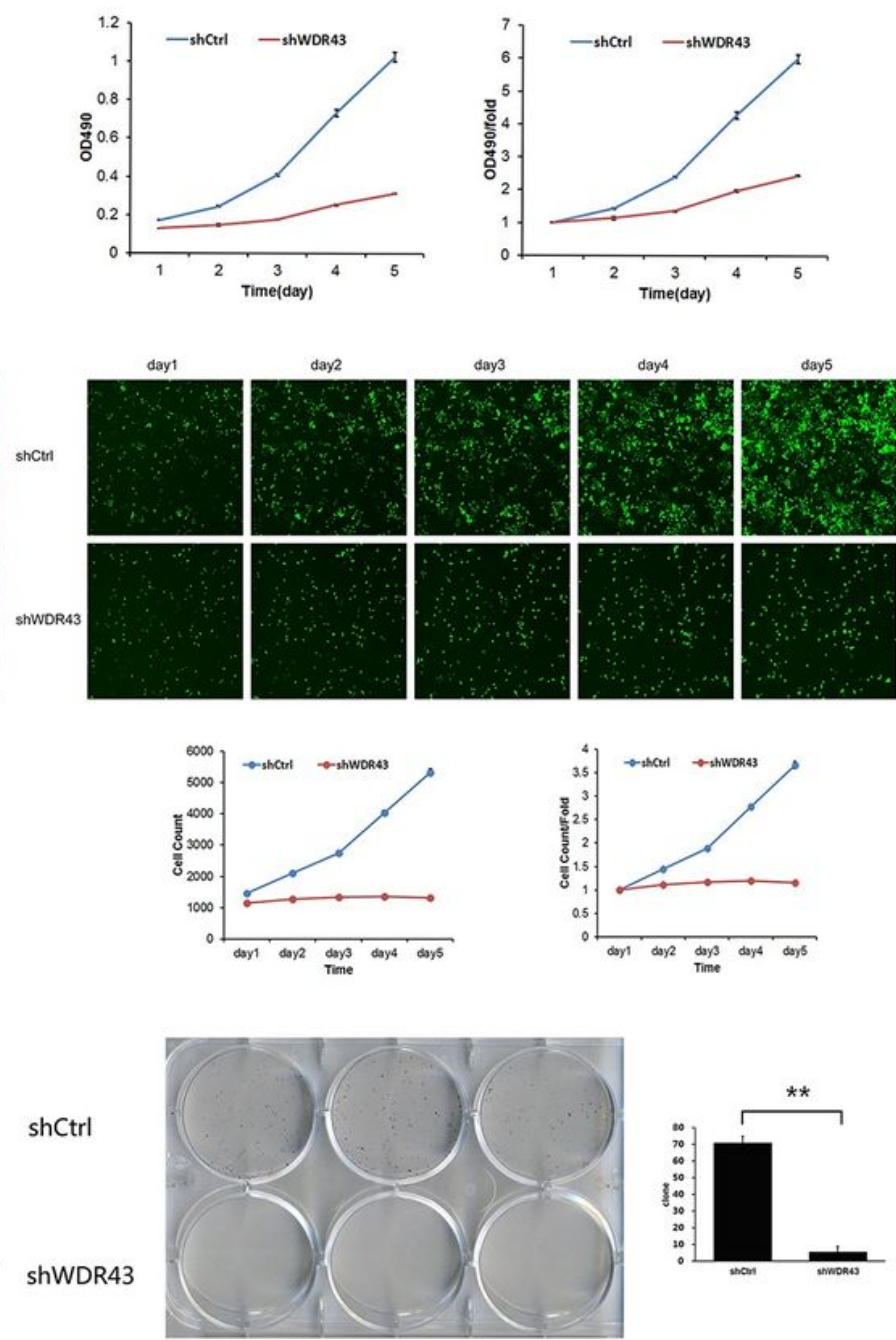

\section{Figure 4}

WDR43 knockdown inhibits the proliferation and colony formation of CRC cells in vitro. Cell proliferation was detected in DLD-1 and HCT116 cells for 5 consecutive days using (a) MTT and (b) Celigo assays. Colony formation assays were performed in both (c) DLD-1 and HCT116 cells. WDR, WD40 repeat; CRC, colorectal cancer; shCtrl, negative control; shWDR43, cells transfected with lentiviral targeting WDR43. 
DLD-1

$\operatorname{shCtrl}$
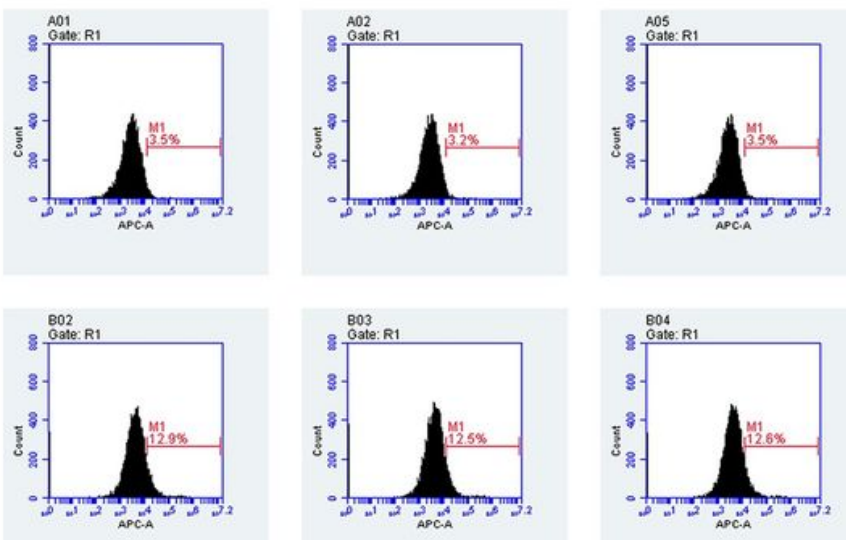

shWDR43

HCT116

shCtrl
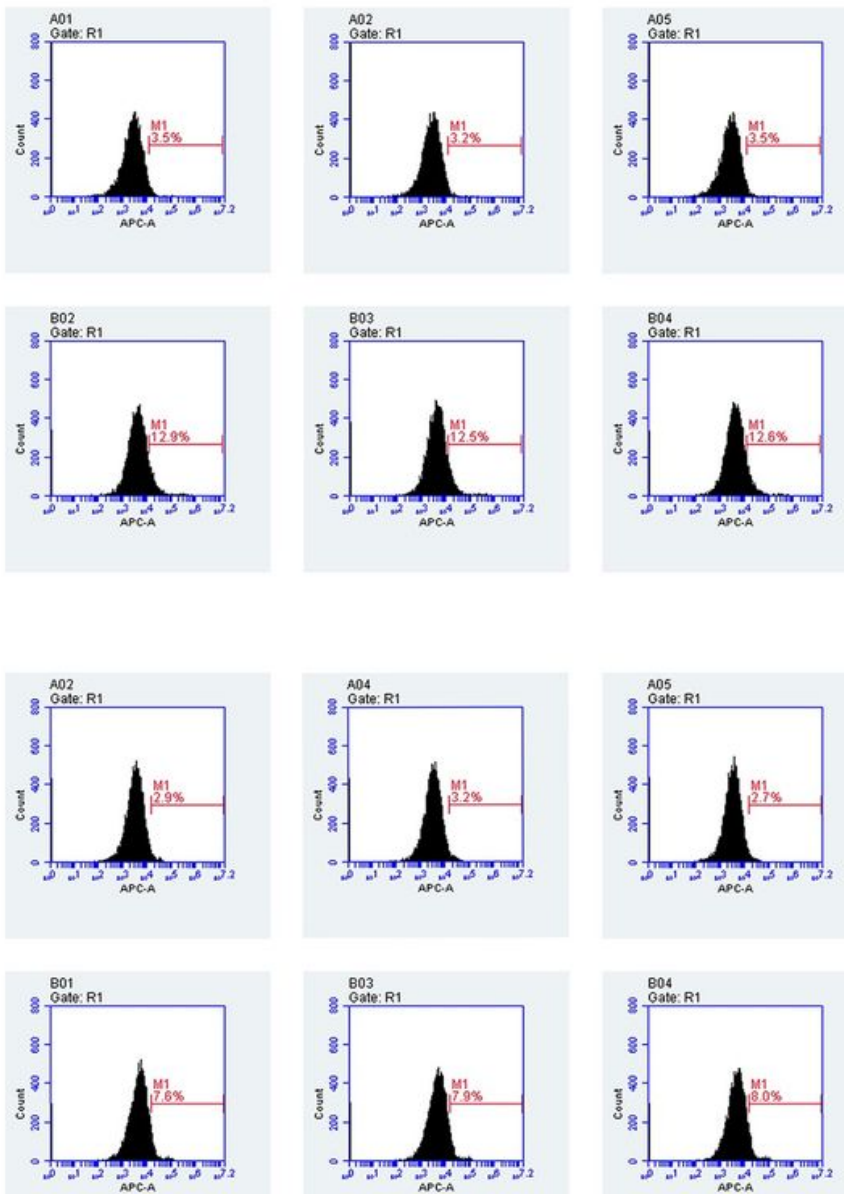
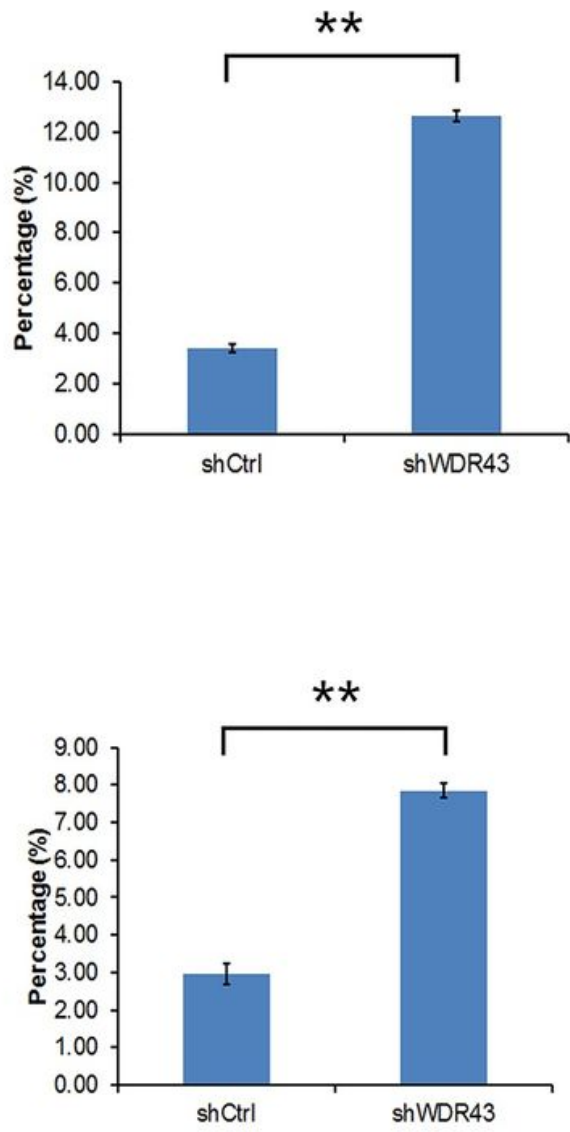

\section{Figure 5}

WDR43 knockdown promotes the apoptosis of CRC cells. The percentage of apoptotic cells was demonstrated by flow cytometry in DLD-1 and HCT116 cell lines following WDR43 knockdown. WDR, WD40 repeat; CRC, colorectal cancer; shCtrl, negative control; shWDR43, cells transfected with lentiviral targeting WDR43.

a DLD-1
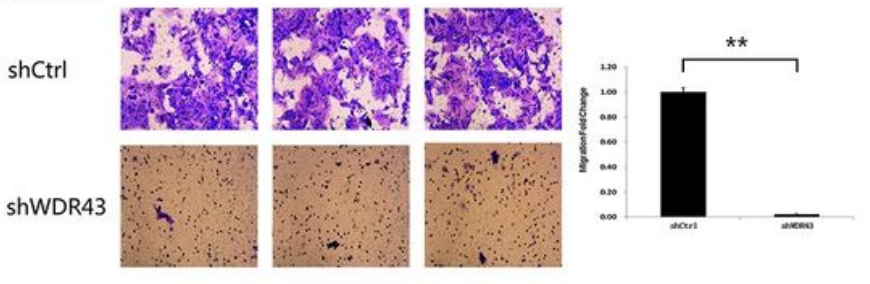

b

shCtrl

shWDR43
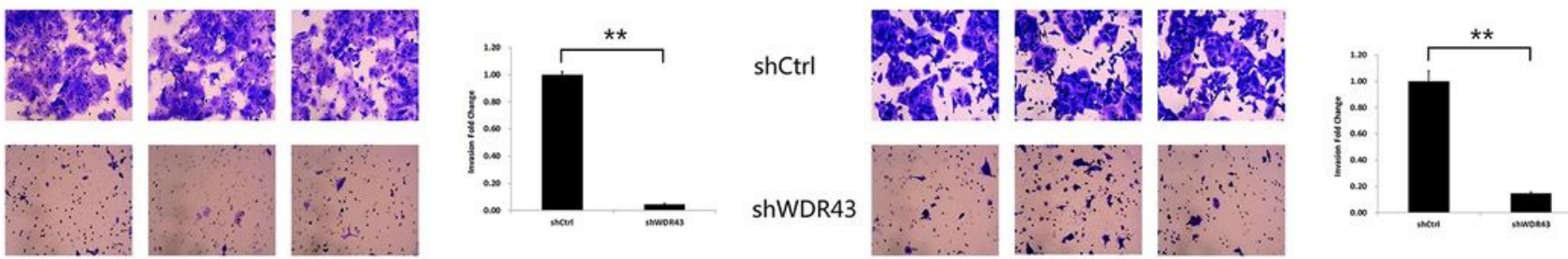
Figure 6

WDR43 knockdown inhibits the migration and invasion of CRC cells. WDR43 knockdown suppressed both cell (a) invasion and (b) migration, as shown by Transwell invasion and migration assays, respectively. WDR, WD40 repeat; CRC, colorectal cancer; shCtrl, negative control; shWDR43, cells transfected with lentiviral targeting WDR43.

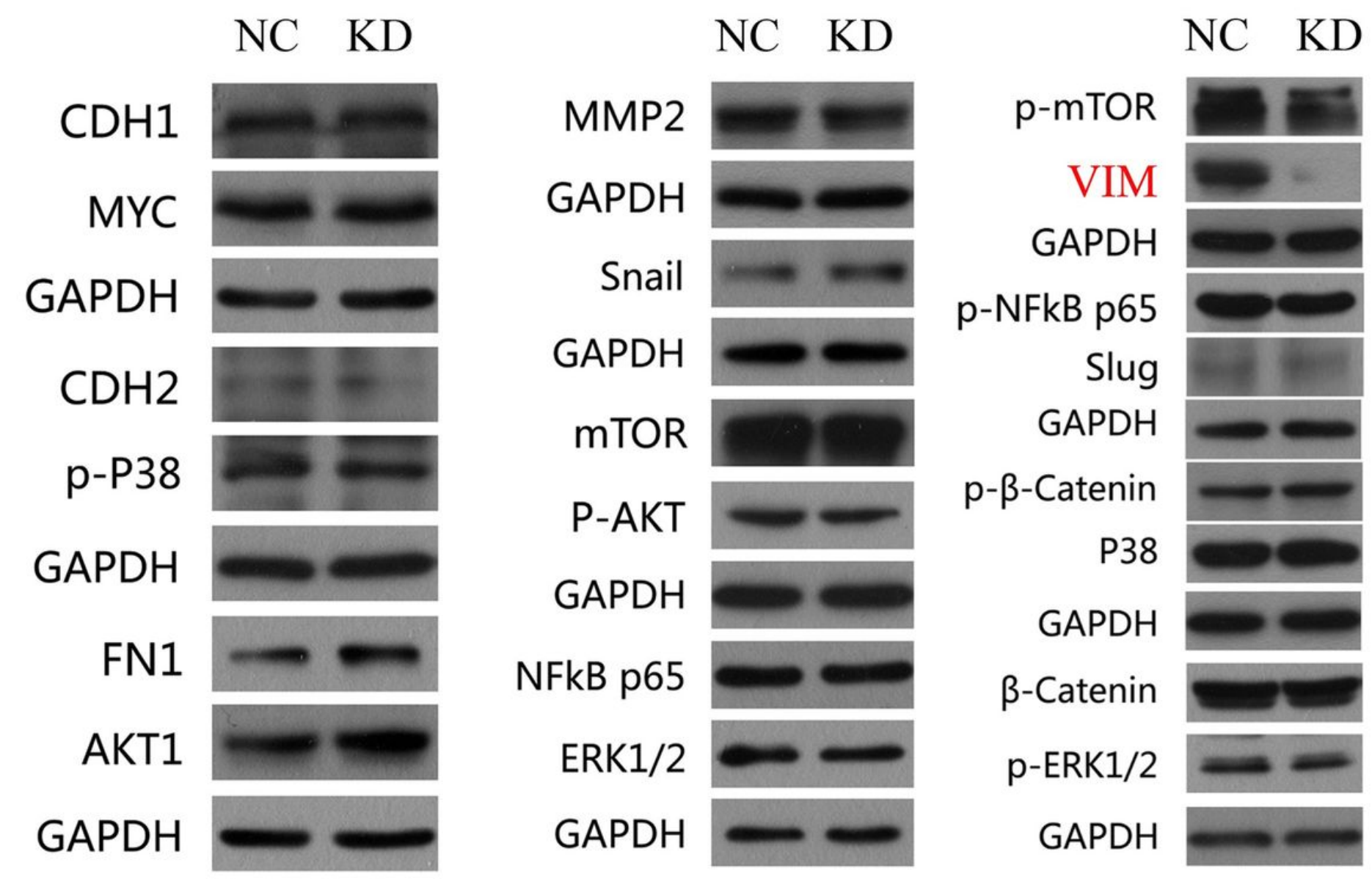

Figure 7

WDR43 knockdown suppresses VIM expression. The levels of several proteins associated with tumor cell proliferation and migration as well as their phosphorylated forms were examined by western blotting, as were those of classical signaling pathway proteins .CDH1, E-cadherin; $\mathrm{CDH} 2$, N-cadherin; FN1, fibronectin 1;(p-)AKT, (phosphorylated-)protein kinase B;MMP-2, matrix metalloproteinase-2;(p-)mTOR, (phosphorylated-)mechanistic target of rapamycin;(p-)NF-KB p65, (phosphorylated-)nuclear factor-kB p65; (p-)ERK1/2, (phosphorylated-)extracellular signal-regulated kinase1/2;VIM, vimentin. NC,negative control; KD, WDR43 knockdown. 
a

b
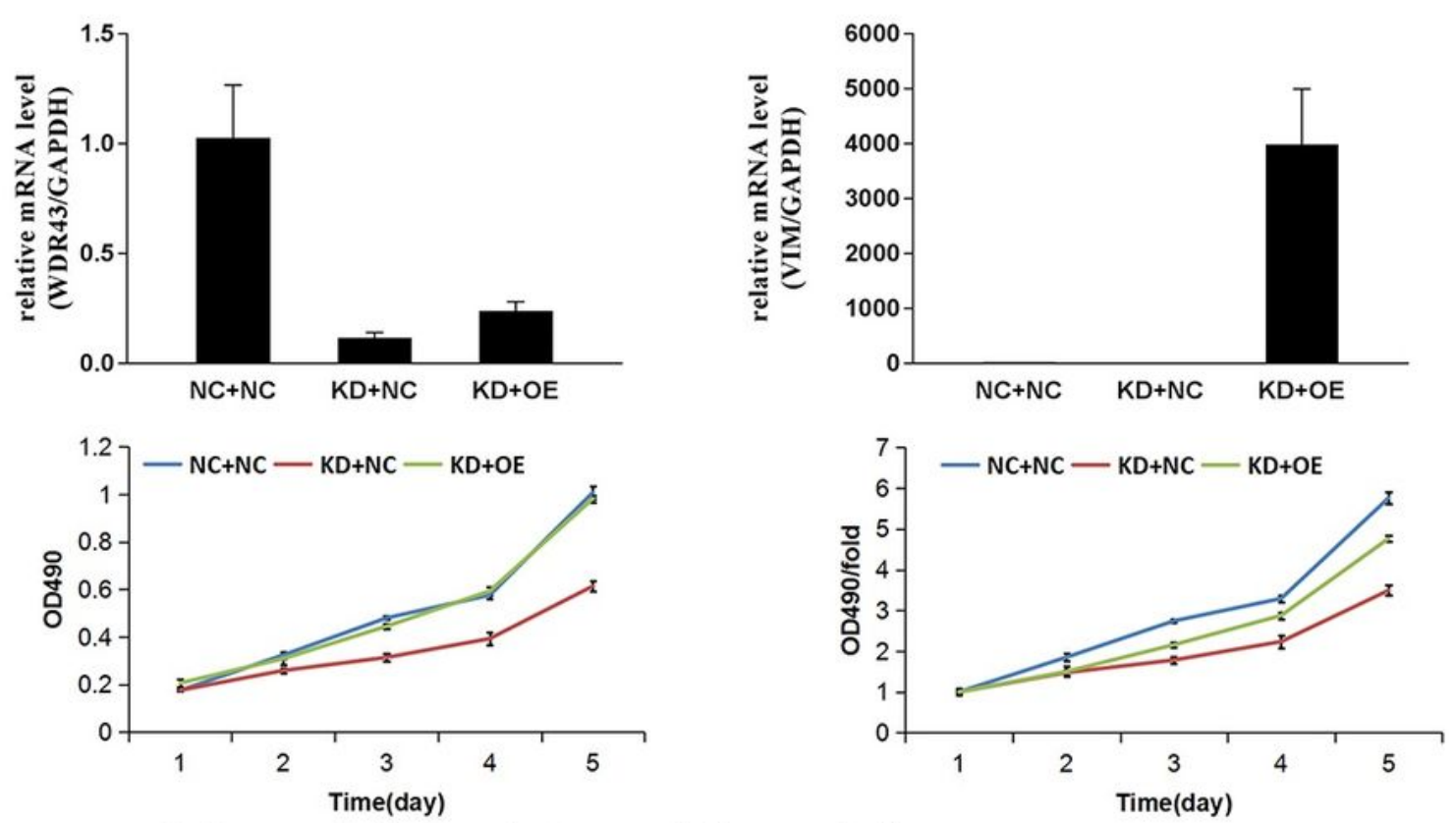

C day 1 day2 day 3 day
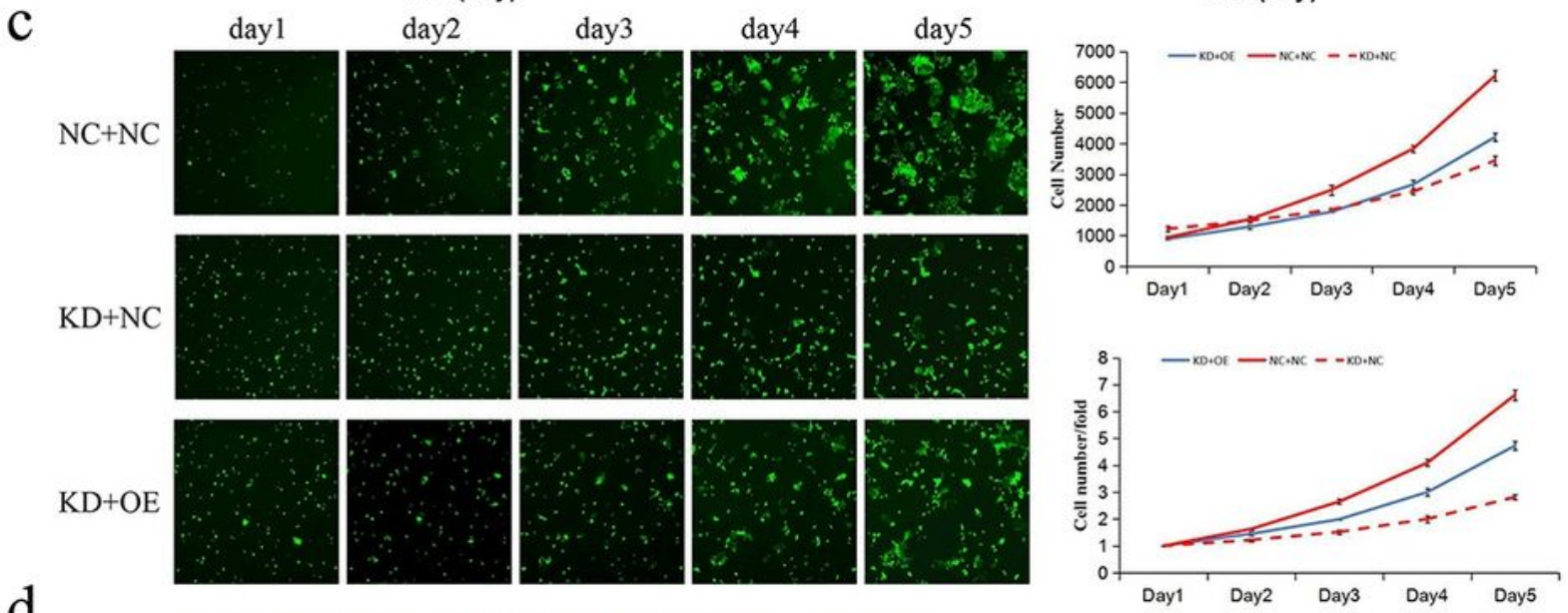

d
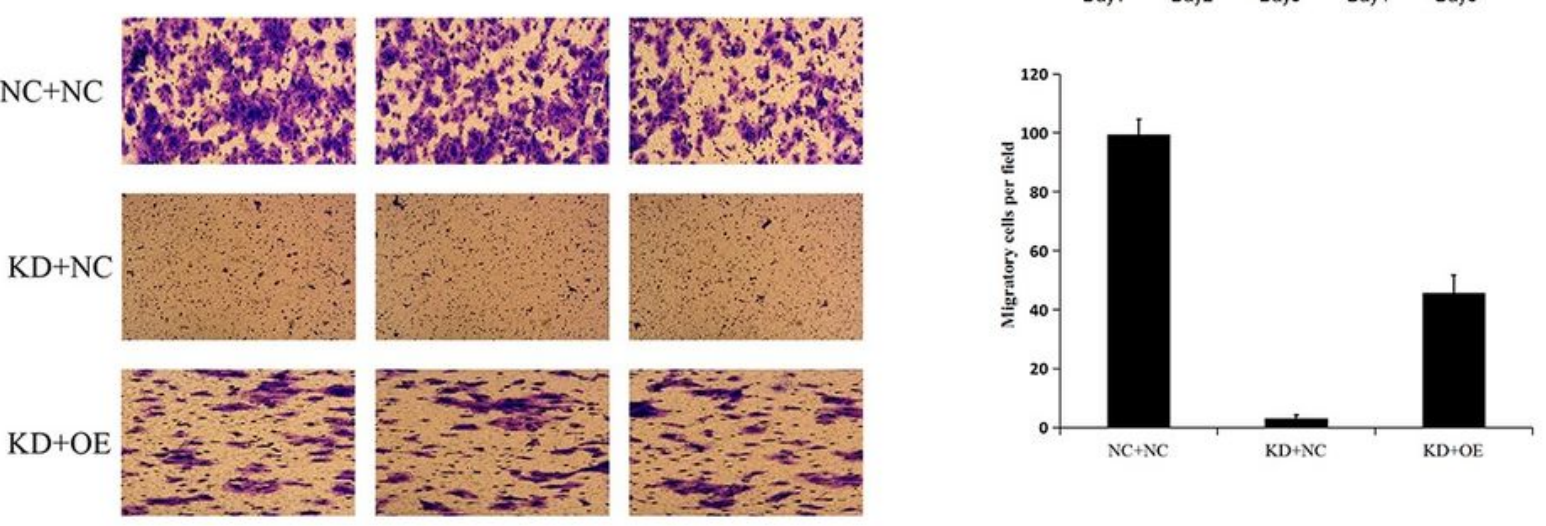

\section{Figure 8}

VIM overexpression partially restores the proliferative and migratory ability of CRC cells following WDR43 knockdown. (a) The transfection efficiency of WDR43 knockdown and VIM overexpression was detected by RT-qPCR. Cell proliferation was detected by (b) MTT and (c) Celigo assays in DLD-1 cells. (d) Transwell assay was also performed in DLD-1 cells. VIM, vimentin; CRC, colorectal cancer; WDR, WD40 repeat; $\mathrm{NC}$,negative control; KD, WDR43 knockdown; OE, VIM overexpression. 
$\mathrm{NC}+\mathrm{NC}$
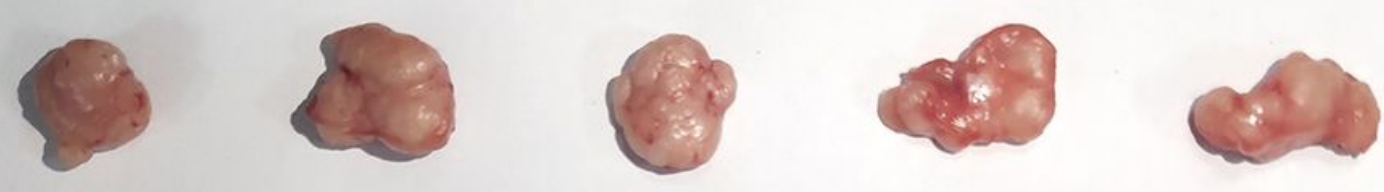

$\mathrm{KD}+\mathrm{NC}$

$\mathrm{KD}+\mathrm{OE}$

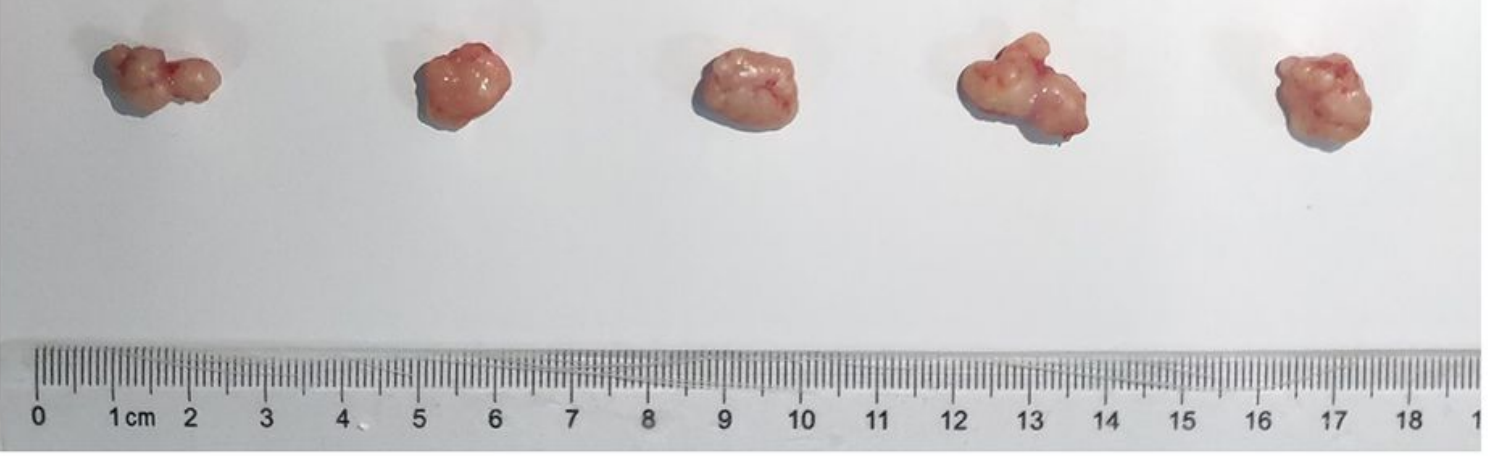

\section{Figure 9}

WDR43 knockdown inhibits the proliferation of CRC cells in vivo. Images of subcutaneous xenografts from mice in the WDR43 knockdown, WDR43 knockdown with VIM overexpression, and NC groups. $\mathrm{n}=5$. WDR, WD40 repeat; CRC, colorectal cancer; VIM, vimentin; NC, negative control; KD, WDR43 knockdown; $\mathrm{OE}, \mathrm{VIM}$ overexpression.

\section{Supplementary Files}

This is a list of supplementary files associated with this preprint. Click to download.

- TableS1.xIsx

- Tables2.xlsx

- Tables3.xls

- Tables4.xls

- Tables5.xls

- Tables6.xls 